\title{
Single Channel Wireless EEG: Proposed Application in Train Drivers
}

\author{
Surya Darma Ridwan ${ }^{*}$, Robert Thompson", Budi Thomas Jap*, Sara Lal", Peter Fischer ${ }^{+}$ \\ *University of Technology, Sydney \\ Broadway NSW 2007, Australia \\ Surya.D.Ridwan@student.uts.edu.au \\ Robert.Thompson@student.uts.edu.au, \\ Budi.T.Jap@uts.edu.au, \\ Sara.Lal@uts.edu.au \\ ${ }^{+}$Signal Network Technology Pty Ltd \\ Lane Cove NSW 2066, Australia \\ p.fischer@telstra.com
}

\begin{abstract}
Electroencephalography (EEG) can be used as an indicator of fatigue. Several studies have shown that slow wave brain activities, delta (0-4 Hz) and theta (4$8 \mathrm{~Hz}$ ), increase as an individual becomes fatigued, while the fast brain activities, alpha $(8-13 \mathrm{~Hz})$ and beta (13-35 Hz), decrease. However, an EEG is a complex piece of equipment that is generally used in laboratory based studies. In order to develop a fatigue countermeasure device for train drivers using EEG, there is a need for a simple and wireless EEG monitor. This paper explains the development of a single channel wireless EEG device.
\end{abstract}

Keywords: EEG, single channel, wireless, prototype development, fatigue detection, train driver

\section{Introduction}

In the late 1800s Richard Caton (1842-1926) first reported the presence of biopotentials on the surface of the human skull [1]. The electroencephalography (EEG), pioneered by Hans Berger, measures the electric potentials on the scalp and provides continuous measure of cortical activity [2, 3]. EEG has played an important role due to its non-invasiveness and the capability of long term measurement in the field of epilepsy, sleeping disorder, fatigue, and various neurological conditions [2-5].

An example of EEG application is in the detection of driver fatigue. Driver fatigue is a process that involves successive episodes of micro-sleeps, where the subject may go in and out of a fatigue state [6]. EEG has been used in many driver studies conducted in the lab and field [5, 7-10], and is one of the most reliable indicators of fatigue [11]. Studies have found that EEG has acceptable test and retest reliability [6, 12-15]. Subsequently, Lal \& Craig [16] proposed that EEG could be used as an indicator of fatigue and proposed driver fatigue algorithms. Later, Jap et al. [17] proposed an EEG-based fatigue algorithm for application in the train driving environment. Lal \& Craig [7] reported increases in slow wave brain activity with driver fatigue. Delta $(0-4 \mathrm{~Hz})$ and theta $(4-8 \mathrm{~Hz})$ activities have been found to increase as one gets fatigued [16]. Jap et al. [17] found significant decreasing trends of alpha (8-13 Hz), and beta (13-35 $\mathrm{Hz}$ ) activities as fatigue increases. Beta activity has also been linked to task performance. As beta level decreases, task performance has also been found to decrease [5].

However, the conventional EEG machine involves attaching the machine to a computer through a data cable, and this introduces some limitations in the applications of EEG monitoring as a fatigue detector [4]. In the actual driving environment, recording EEG data using a conventional EEG machine may not be feasible, because EEG is a complicated piece of technology for laboratory environment setup. The cables from the EEG machine may be intrusive and hinder the driver. Therefore, a simple, portable, and wireless EEG is required for application in fatigue monitoring.

This technical chapter proposes the design of a single channel wireless EEG device that is suitable for fatigue detection in train drivers. The EEG measurement was designed for bipolar montage recording. The recording in bipolar montage is obtained from the difference between two active electrodes that are adjacent to each other on a particular brain site, e.g. frontal site [18]. The EEG electrodes are aimed to be placed inside a headband at 
either the frontal or temporal sites, according to the 1020 electrode placement standard, in order to achieve uniformity in the interpretation of recorded results [19, 20].

The following sections will describe the development of a single channel wireless EEG prototype, and will present a few results on EEG recordings obtained from the prototype.

\section{Hardware Development}

The amplitude of the EEG signal depends on how synchronous the activities of the underlying neurons are [21]. The human EEG signal ranges in frequency from $0.5-100 \mathrm{~Hz}$, with amplitudes of $1-300 \mu \mathrm{V}$ measured at the surface of the skull $[3,20,22]$. These signal characteristics have inherent challenges to their measurement. Firstly, $50 \mathrm{~Hz}$ noise is present within the
EEG frequency range. Significant electrical noise is present at this frequency in most environments. The very low frequency EEG signal makes it susceptible to $50 \mathrm{~Hz}$ noise. The signal may further be corrupted by impedance imbalance at the skin-electrode interface, electrode half-cell potential, and movement artifacts [3].

A prototype wireless EEG system has been developed in order to extract EEG signal from human subjects, and to be analysed for fatigue detection. The hardware module consists of transmitter and receiver ends. While the receiver end is digital, the transmitter end consists of analog and digital sections. A block diagram of the system is depicted in Figure 1. The prototype is designed to operate with a 6-volt (V) battery. A voltage divider and voltage follower are used to obtain a +/-3 V supply.

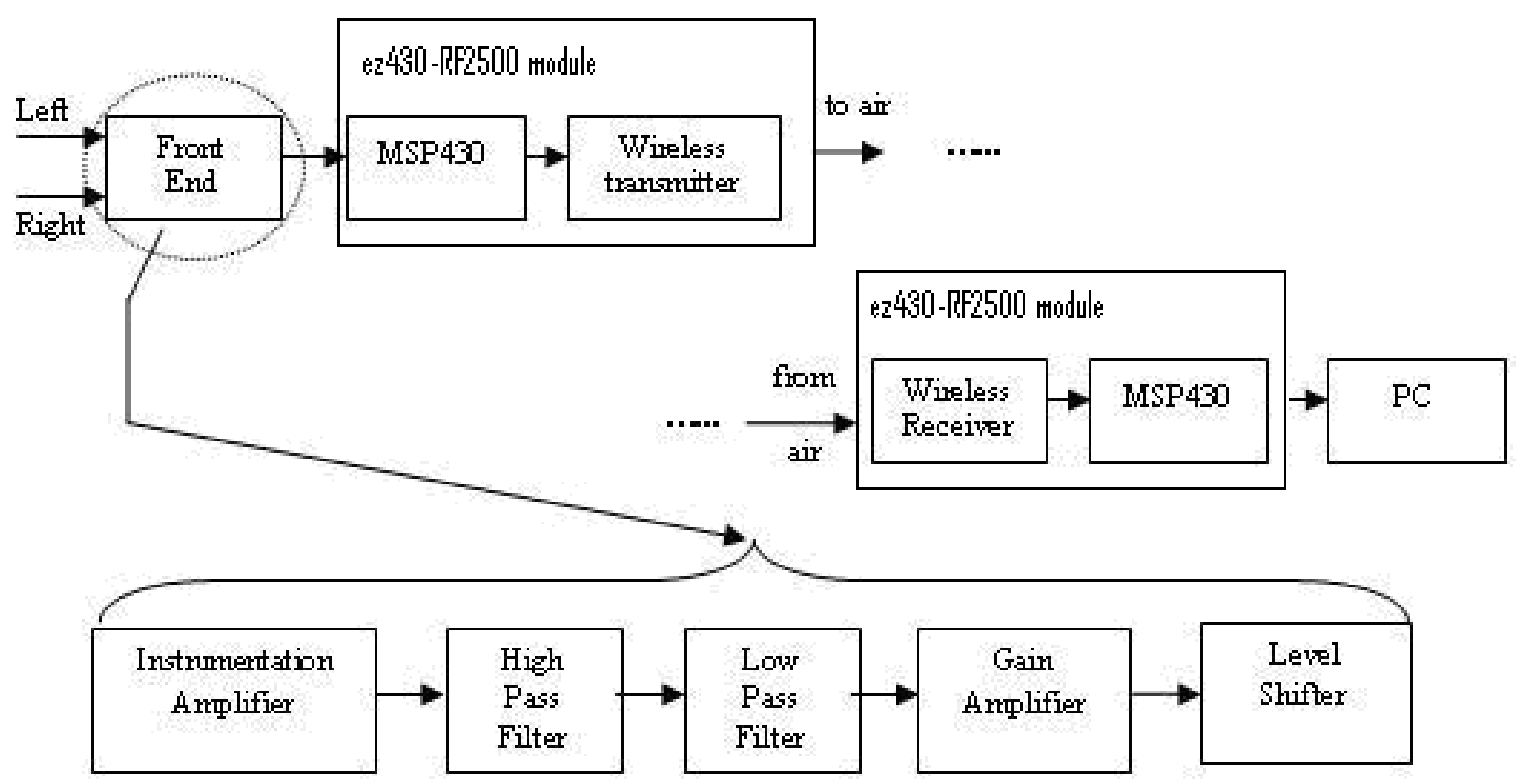

Figure 1 Block diagram of the EEG system prototype

\subsection{Electrodes}

The electrodes used for this system is the MLA2503 - Shielded Lead Wires Snap-On electrodes (ADInstruments, Australia). The lead wires are 98 centimetres in length with 4 millimetres snap-on connectors used with $3 \mathrm{M}$ Red Dot $\mathrm{Ag} / \mathrm{AgCl}$ (silver/silver-chloride) monitoring electrode (3M, USA). An adequate amount of conductive gel is applied on the electrodes prior to attaching them to the subject. One of the advantages of using the $\mathrm{Ag} / \mathrm{AgCl}$ electrodes is the superior performance in term of lowfrequency noises [23].

\subsection{Analog Front End}

The subject is connected to the front end of the transmitter through three EEG electrodes. The front end is mainly the analogue electronics, which consists of an instrumentation amplifier followed by signal amplification and conditioning stages (i.e. direct current (DC) restorator, gain amplifier, high-pass and low-pass filters, variable attenuation and signal level shifter). The front-end schematic is shown in Figure 2. 


\subsubsection{AD620 Instrumentation Amplifier}

The instrumentation amplifier is used to amplify the difference of the signal from the left and right EEG electrodes, and simultaneously reject common mode noise at both inputs. The AD620 instrumentation amplifier (Analog Devices, USA) was the instrumentation amplifier chosen for this design as it offers low power and high Common Mode Rejection Ratio (CMRR). Without a high CMRR, common mode voltages, which are typically $1 \mathrm{~V}$ [24], will get amplified and prevent the EEG signal from being recovered. The CMRR ratio of AD620 is in the range of 90 to $110 \mathrm{~dB}$ for Gain $\mathrm{G}=5$ in the frequency range from $\mathrm{DC}$ to $60 \mathrm{~Hz}$ [25]. Another reason to choose AD620 as the preamplifier stage is due to its low voltage noise $(9 \mathrm{nV} / \sqrt{\mathrm{Hz}})$ and low input current noise $(0.1 \mathrm{pA} / \sqrt{ } \mathrm{Hz})$.

In the subsequent stages, LMC6484 (low-power Operational Amplifier (op-amp), National Semiconductor, USA) Integrated Circuits (ICs) are used to implement all signal conditioning circuitry. The LMC6484 has low-power consumption making it suitable for portable battery powered applications.
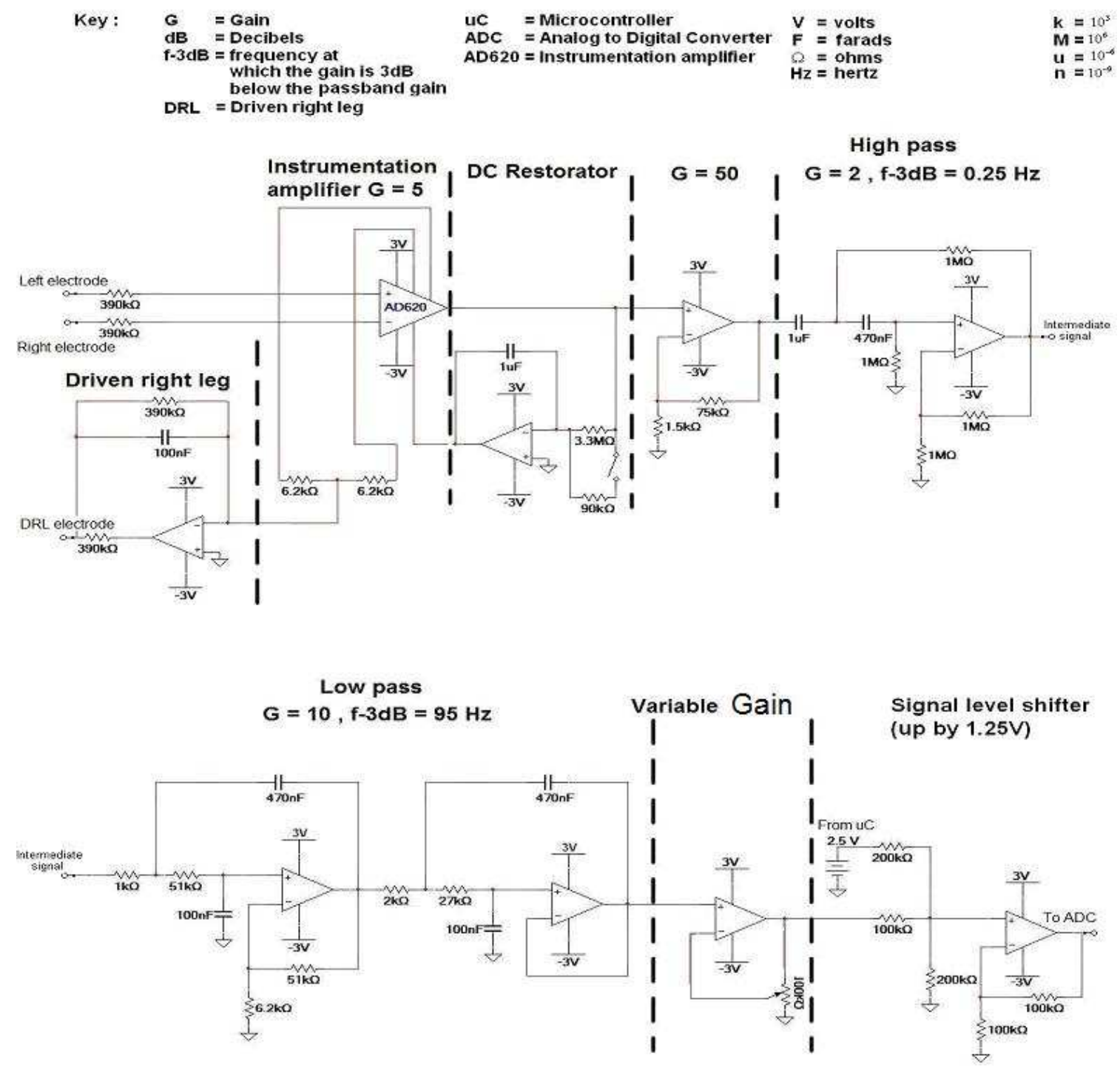

Figure 2 Schematics of the EEG Front End 


\subsubsection{Restorator}

The DC restorator circuit is used to eliminate DC offset that would otherwise saturate the op-amps subsequent to the instrumentation amplifier. The DC restorator is implemented by using an op-amp in the feedback loop of the AD620. The DC restorator has two settings, namely the 'monitor', and the 'quick restore'. The 'quick restore' setting can be used to speed up the recovery of the output if it becomes saturated [3]. The model in Figure 3 was used to design the DC restorator.

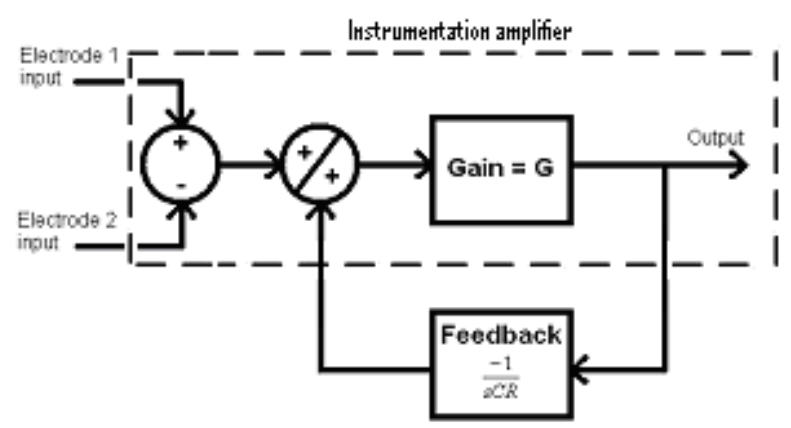

Figure 3 DC Restorator Model

\subsubsection{High Pass and Low Pass Filters}

The high-pass and low-pass filters are used to cutoff the signal with frequency components below 0.25 $\mathrm{Hz}$ and above $95 \mathrm{~Hz}$. The high pass filter is implemented by using a $2^{\text {nd }}$-order Sallen-Key filter [26]. It attempts to eliminate the DC potential present at the electrode. The low pass filter is implemented by using two $2^{\text {nd }}$-order Sallen-Key filter stages. Therefore it is effectively a $4^{\text {th }}$-order filter. Refer to Figure 4 for the frequency response of the high pass and low pass filters.

\subsubsection{Driven Right Leg}

The Driven Right Leg (DRL) is incorporated in the design to reduce common-mode noise, such as $50 \mathrm{~Hz}$ interference unavoidably coupled into the subject and electrodes. The name, Driven Right Leg, has been preserved from its first use in electrocardiography (ECG) equipment [27], although this electrode is not connected to the subject's right leg for EEG recordings. As shown in Figure 2, the DRL is a feedback circuit similar to the DC restorator. It feeds the inverse of the common-mode voltage back to the human subject, which acts to reduce the common-mode noise present at the active electrodes [27].

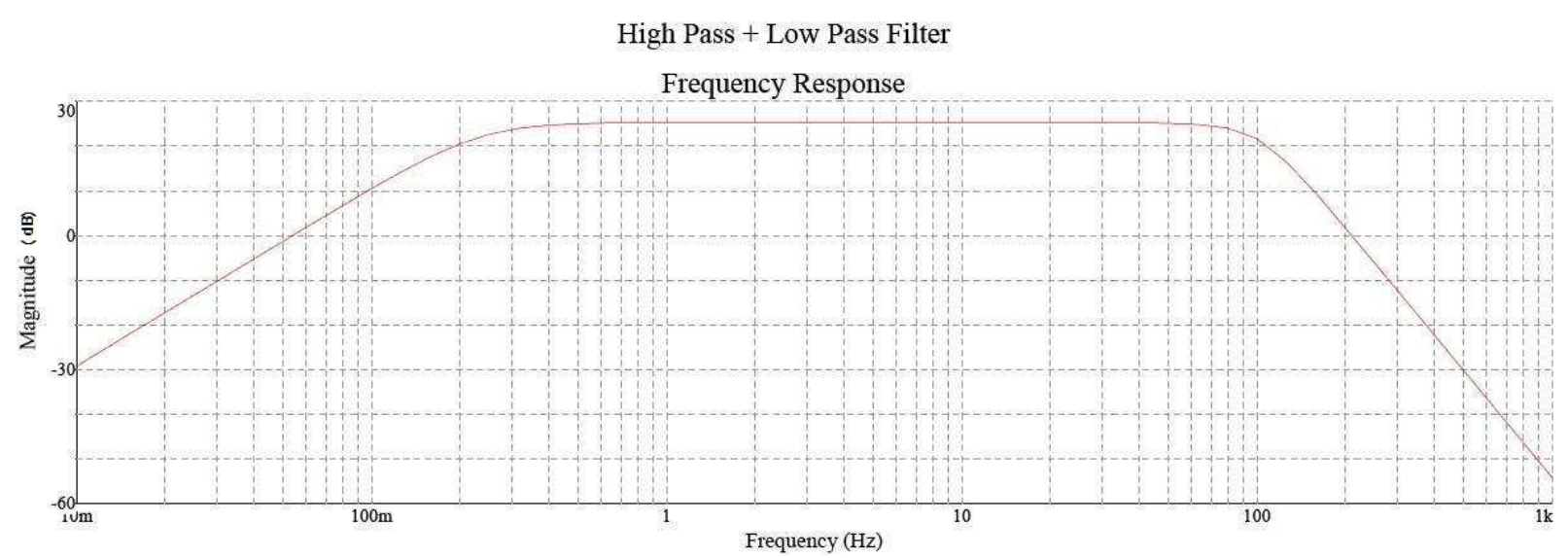

Figure 4 Frequency response of high pass and low pass filters $(\mathrm{dB}=$ decibel; $\mathrm{Hz}=\mathrm{Hertz})$

\subsection{MSP430 and Wireless Modules}

The first prototype of the digital section utilises the eZ430-RF2500 development tool (Texas Instruments, USA). It contains both the MSP430 microcontroller and CC2500 wireless module (see Figure 1). The microcontroller is suitable for low power operation as it only consumes 0.7 microAmps (uA) while in standby mode. In addition, it processes 16-million instructions per second, and is equipped with two 10bit Analog-to-Digital (A-D) converters with maximum sampling rates of 200 Kilo samples per second.

The communication protocol running on CC2500 is SimpliciTI, which is a dedicated low data rate protocol for low power applications. The wireless transmission utilises the 2.4 Giga Hertz (GHz) Industrial, Scientific and Medical (ISM) frequency [28]. 
At the transmitter end, the microcontroller takes the output of the analog front end and performs the A-D conversion. A fatigue detection algorithm could be implemented in the microcontroller at the transmitter end to detect fatigue before the digitised signal is transmitted to the CC2500 module of the receiver end.

At the receiver end, the same eZ430-RF2500 development board, which is used at the transmitter end, is connected to a PC via a USB slot.

\section{Experiments and Results}

Figure 5 shows the prototype that has been developed for the project. The EEG signal was transmitted from the TI MSP430 Wireless transmitter to the computer. To aid the experiment, a testing program was built (using LabView 8.2, National Instruments, USA) in order to display the received signal at the receiver end. The development prototype was tested on a human subject.

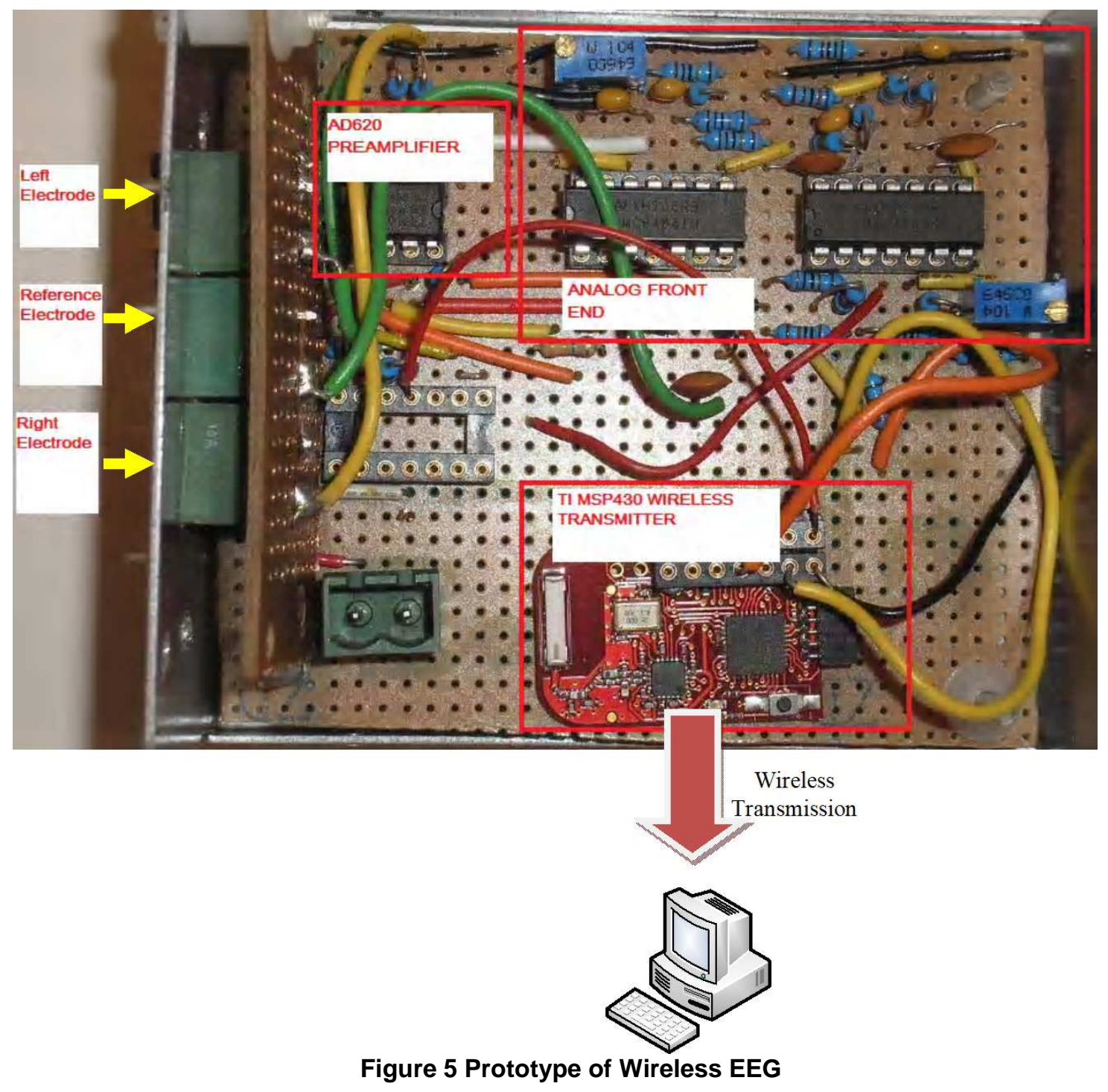

Firstly, a $10 \mathrm{~Hz}, 100 \mathrm{mV}$ sine-wave was used to verify the accuracy of the Wireless EEG prototype. The amplitude of the sine wave was further scaled down by means of voltage divider into a few hundred microvolts before being pumped into the preamplifier stage.
Figure 6 displays the received sine wave pattern at the receiver's end, which proves the accuracy of the Wireless EEG prototype. 


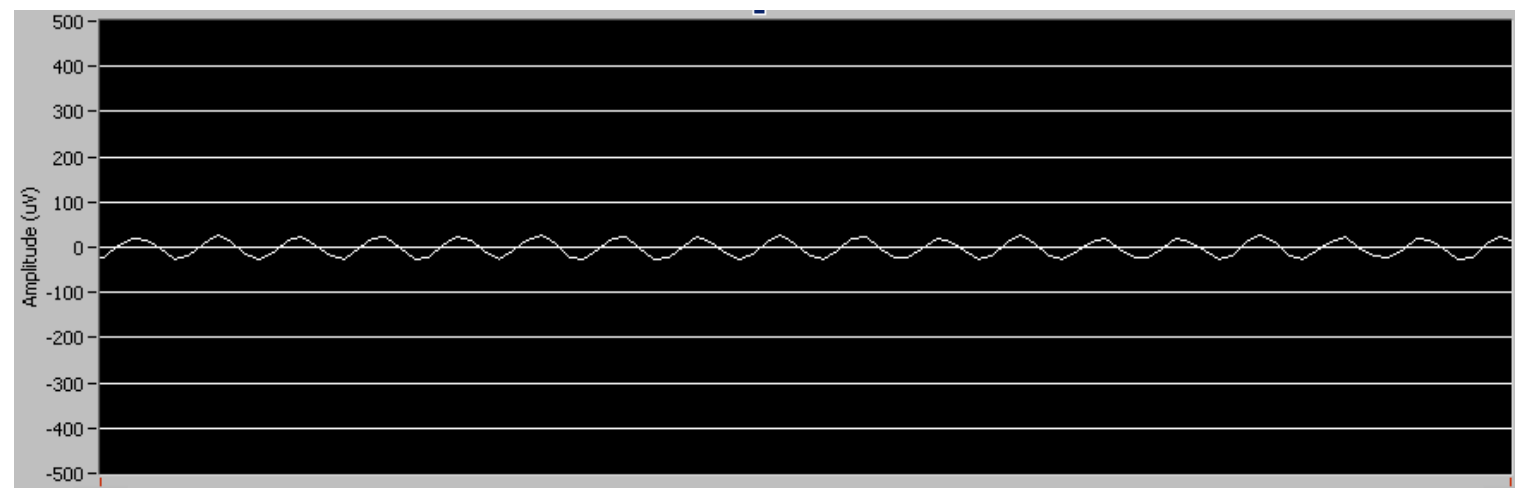

Figure 6 Display of the received sine wave from the wireless transmission

The development prototype was then tested on a human subject to capture ECG signal (heart beat) by connecting 3 electrodes from the device to the left wrist, right wrist and right leg of the subject. The subject was asked to remain seated and stop moving in order to avoid movement artifacts. With the aid of the testing program, the device was able to successfully capture the PQRS complexes of the ECG signal from the subject, as shown in Figure 7.

Finally, the circuit gain was adjusted to allow sufficient amplification for acquiring a typical EEG signal. The prototype was then used to capture the EEG signal from electrodes placed on the frontal lobe of the head. This was performed by connecting a pair of shielded electrodes to the pre-frontal brain sites (FP1 and FP2) and the DRL electrode was connected to the ear lobe of the subject. The subject was again asked to remain seated and stay still to avoid movement artifacts. Figures 8 and 9 depict the EEG signal during eyes-open and eye-closure, respectively. In Figure 8, it was noted that an eye blink would cause an artifact in the EEG waveform. In contrast, the EEG reading with the eyes closed in Figure 9 showed fewer artifacts.

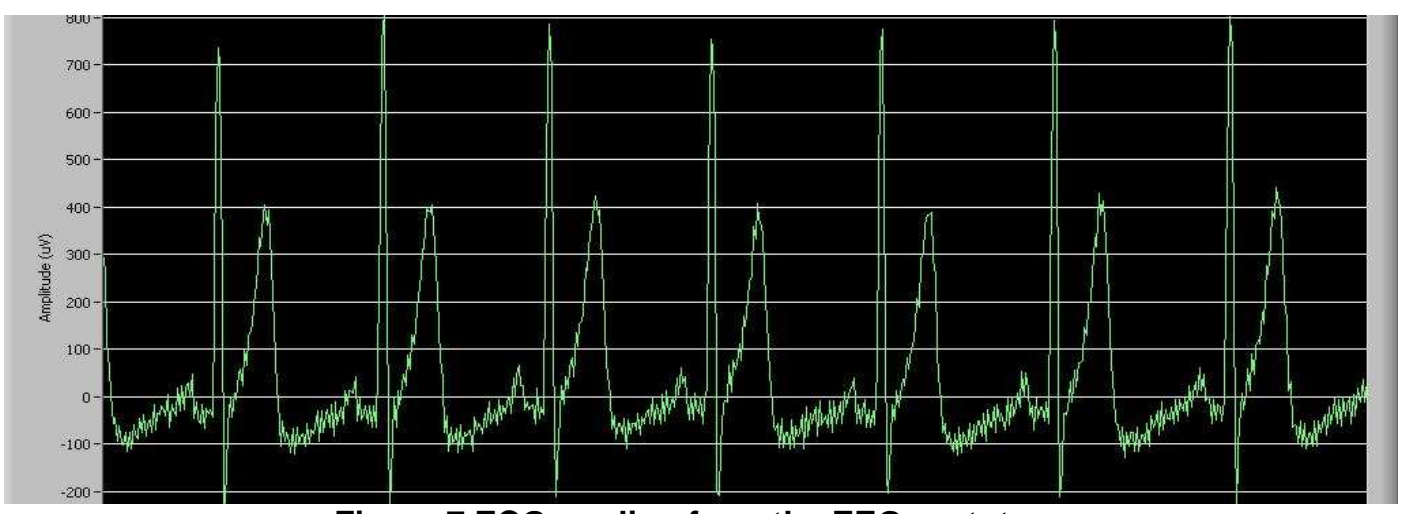

Figure 7 ECG reading from the EEG prototype

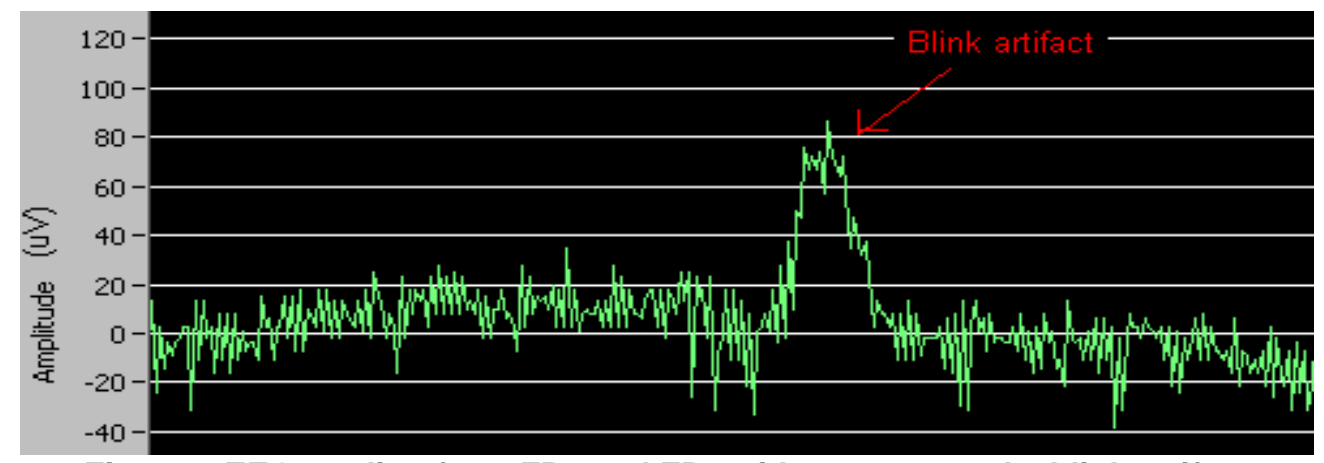

Figure 8 EEG reading from FP1 and FP2 with eyes opened - blink artifact 


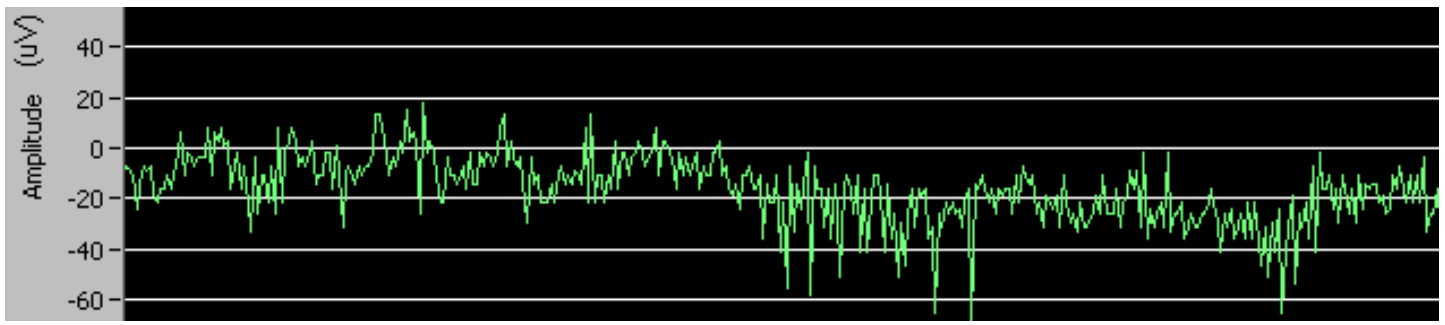

Figure 9 EEG reading from FP1 and FP2 with eyes closed

\section{Discussion and Future Work}

The area of safety in transportation has received attention due to a high number of accidents caused by driver fatigue [29]. Railway is a relatively safe transportation means. The number of train accidents and injuries in Australia is relatively low, when compared to road and aviation industries [30]. However, given the fact that the travelling speed of a train is relatively faster than a car, a train carriage is much heavier than a car, and that trains' braking time is slower than a car (about $1.1 \mathrm{~m} / \mathrm{s}^{2}$ ) [31], the impact that it creates in an accident causes more injuries due to a usually large numbers of passengers involved, destruction to the surrounding area and emotional and financial costs to community. Unlike the driver of a car who can swerve his or her vehicle to avoid collisions, a train driver can only apply the emergency brake, sound the horn and hope that the train will stop in time before the collision [32, 33], and if the driver is in a fatigue state even these safety measures may fail.

In the train driving environment, human errors caused by fatigue contributes to about $75 \%$ of accidents [34, 35], and research has shown that driving while fatigued is just as dangerous as driving with a blood alcohol concentration of $0.05-0.1 \%$ [36]. Train drivers are required to work in a 24-hour irregular and rotating shifts, including weekends [37], and irregular shifts are related to increased fatigue level and have been correlated to higher risk of fatigue-related accidents [38]. Therefore, there is a need for an automated and non-intrusive fatigue countermeasure device for drivers in this environment.

Electroencephalography has been shown to be a reliable fatigue indicator [11]. Several studies have already investigated the efficacy of EEG as a fatigue indicator. Lal and Craig [7], Lal et al. [16], Jap et al. [39] and Eoh et al. [5] proposed fatigue algorithm derived from the four EEG frequency bands. Tietze [40] explored detection of fatigue using the occurrence of 'alpha-spindles', which consisted of short periods of high amplitude alpha activity, and could be visually detected. Others have proposed the use of neural network, wavelet transform, or Independent Component Analysis (ICA) algorithm to detect fatigue [41-44].

However, the complexity of an EEG system makes it difficult to deploy it as a fatigue countermeasure device in the actual train driving environment [45]. Simple and wireless EEG electrodes that can be worn by drivers while driving need to be developed, before fatigue countermeasures using EEG will become viable. These simple EEG measurement systems will also need to be able to reduce signal noise that is received from surrounding environment. Since drivers are also dynamic human beings, they may use mobile phones, which introduce high-frequency noise into the EEG recording. The noise may affect the recordings and reduce the accuracy of the device to detect fatigue.

The current chapter proposes the prototype development of a simple single-channel EEG device that transmits wirelessly to a computer. Bipolar montage has been used for the analog front-end, by amplifying the difference of the EEG signals from the left and the right active electrodes, and the DRL electrode has been implemented to reduce the commonmode noise that are present at the active electrodes. The eZ430-RF2500 development tool has been utilized to transmit EEG signals wirelessly.

The simulation result of the device shows that noise is present when recording ECG or EEG (Figures 7 to 9). Electrical noise, such as $50 \mathrm{~Hz}$ interference, has been filtered by the high- and low-pass filters implemented in the design. The noise that is captured in the recording may be due to insufficient contact being established between the electrodes and the skin, or subject's involuntary movements.

For the current prototype development of the wireless EEG, the device transmitted the sampled EEG signal from the analog front end to the computer. The computer then processed the received EEG signal, transformed it to frequency domain using Fast Fourier Transform (FFT) computation, and detected whether or not the person is in a fatigued state. The FFT 
computation may be able to be incorporated into the microcontroller. However, the on-board memory of the MSP430 microcontroller was considerably small to be able to perform effective Fast Fourier Transform (FFT) computation. Future work may incorporate external memory into the microcontroller design to address the current issue. Fatigue detection may also be performed within the wireless EEG device if the device has enough memory capacity, and fast computation speed.

The current prototype utilized the eZ430-RF2500 development tool from Texas Instruments, USA, since the on-board microcontroller was also acting as a transceiver. Other wireless technologies, such as Bluetooth or ZigBee, may also need to be explored to discover a better quality device. Bluetooth is a nonexpensive device with short-range data communication, low data rate and low power consumption [46, 47]. Data can be transmitted to a distance of up to $10 \mathrm{~m}$ with low power usage of less than 1 Milliwatt $(\mathrm{mW})$ [48, 49]. ZigBee protocol is similar to Bluetooth protocol with some minor differences in functionalities, because this protocol was developed to provide improvements to Bluetooth technology [50]. However, when compared with Bluetooth, some advantages and disadvantages still exist in ZigBee technology. The coverage area for ZigBee is comparatively larger with a radius of $70 \mathrm{~m}$, with 64,000 nodes that can be connected to a master device, compared to only 7 slaves for Bluetooth devices [50]. However, the data rate is reduced to approximately $250 \mathrm{Kbps}$, and ZigBee is only suitable for small data packet transmissions, while Bluetooth is capable to transmit large data sets, audio, graphics, or files [50]. Other types of wireless technologies may also be explored in the future development.

The current wireless EEG device has to be miniaturized in the future work in order to fit into a headband or a cap, as shown in Figure 10. Such design implementation will be suitable for train driving environment, since the wireless EEG will be nonintrusive in a driving situation.

Fatigue detection is one of the areas that wireless EEG can be utilized. Applications of wireless EEG can be expanded to other areas, such as simple monitoring of the state of an individual in the daily life environment outside laboratories or hospitals. However, for every development of wireless EEGs, the design of the device has to conform with the safety requirements according to the IEC 60601-1:1988 (safety standard of medical electrical equipment). The next stage of the prototype development will need to address this safety issue.

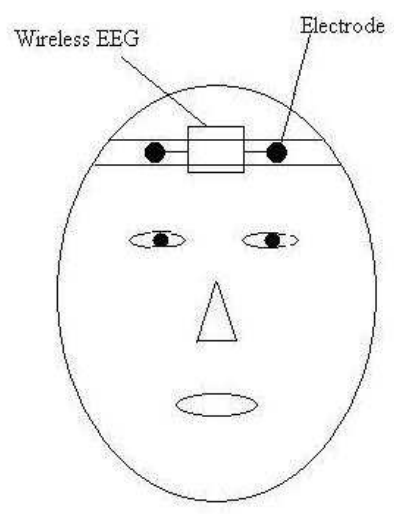

\section{Figure 10 Proposed wireless EEG headband for the development of a fatigue countermeasure device}

\section{Acknowledgement}

The research was supported by an ARC Linkage grant Australia (LP0560886) and by SENSATION Integrated Project (FP6-507231) co-funded by the Sixth Framework Programme of the European Commission under the Information Society Technologies priority.

\section{References}

[1] L. F. Haas, "Hans Berger (1873-1941), Richard Caton (1842-1926), and electroencephalography. (Neurological Stamp)," Journal of Neurology, Neurosurgery and Psychiatry, vol. 74, p. 9, 2003.

[2] W. De Clercq, B. Vanrumste, J.-M. Papy, W. Van Paesschen, and S. Van Huffel, "Modeling Common Dynamics in Multichannel Signals With Applications to Artifact and Background Removal in EEG Recordings," IEEE Transactions on Bioimedical Engineering, vol. 52, pp. 2006- 2015, 2005.

[3] J. J. Carr and J. M. Brown, Introduction to Biomedical Equipment Technology, 4th ed. New Jersey, USA: Prentice Hall, 2001.

[4] R. Lin, R.-G. Lee, C.-L. Tseng, Y.-F. Wu, and J.A. Jiang, "Design and Implementation of Wireless Multi-channel EEG Recording System and Study of EEG Clustering Method," Biomedical Engineering - Applications, Basis \& Communications, vol. 18, pp. 276-283, 2006.

[5] H. J. Eoh, M. K. Chung, and S.-H. Kim, "Electroencephalographic study of drowsiness in simulated driving with sleep deprivation," International Journal of Industrial Ergonomics, vol. 35, pp. 307-320, 2005. 
[6] S. K. L. Lal and A. Craig, "Reproducibility of the spectral components of the electroencephalogram during driver fatigue," International Journal of Psychophysiology, vol. 55, pp. 137-143, 2005.

[7] S. K. L. Lal and A. Craig, "Driver Fatigue: Electroencephalography and Psychological Assessment," Psychophysiology, vol. 39, pp. 313321, 2002.

[8] T. Åkerstedt, G. Kecklund, and A. Knutsson, "Manifest Sleepiness and the Spectral Content of the EEG during Shift Work," Sleep, vol. 14, pp. 221-225, 1991.

[9] G. Kecklund and T. Åkerstedt, "Sleepiness in long distance truck driving: an ambulatory EEG study of night driving," Ergonomics, vol. 36, pp. 10071017,1993

[10] O. G. Okogbaa, R. L. Shell, and D. Filipusic, "On the investigation of the neurophysiological correlates of knowledge worker mental fatigue using the EEG signal," Applied Ergonomics, vol. 25, pp. 355-365, 1994.

[11] P. Artaud, S. Planque, C. Lavergne, H. Cara, P. de Lepine, C. Tarriere, and B. Gueguen, "An on-board system for detecting lapses of alertness in car driving," in 14th E.S.V. Conference, Session 2 Intelligent Vehicle Highway System and Human Factors, Munich, Germany, 1994.

[12] A. J. Tomarken, R. J. Davidson, R. E. Wheeler, and L. Kinney, "Psychometric properties of resting anterior EEG asymmetry: temporal stability and internal consistency," Psychophysiology, vol. 29, pp. 576- 592, 1992.

[13] V. E. Pollock, L. S. Schneider, and S. A. Lyness, "Reliability of topographic quantitative EEG amplitude in healthy late-middle-aged and elderly subjects," Electroencephalography and Clinical Neurophysiology, vol. 79, pp. 20-26, 2002.

[14] T. Gasser, P. Bacher, and H. Steinberg, "Test-retest reliability of spectral parameters of the EEG," Electroencephalography and Clinical Neurophysiology, vol. 60, pp. 312-319, 1985.

[15] T. Gasser, L. Sroka, and J. Möcks, "The transfer of EOG activity into the EEG for eyes open and closed," Electroencephalography and Clinical Neurophysiology, vol. in press, 1985.

[16] S. K. L. Lal, A. Craig, P. Boord, L. Kirkup, and H. Nguyen, "Development of an algorithm for an EEG-based driver fatigue countermeasure," Journal of Safety Research, vol. 34, pp. 321-328, 2003.

[17] B. T. Jap, S. Lal, P. Fischer, and E. Bekiaris, "Using Spectral Analysis to Extract Frequency Components from Electroencephalography: Application for Fatigue Countermeasure in Train Drivers," in The 2nd International Conference on Wireless Broadband and Ultra Wideband Communications (AusWireless 2007) Sydney, Australia, 2007.

[18] J. M. Stern and J. Engel, Altas of EEG Patterns. USA: Lippincott Williams \& Wilkins, 2005.
[19] H. H. Jasper, "Report of the committee on methods of clinical examination in electroencephalography : 1957," Electroencephalography and Clinical Neurophysiology, vol. 10, pp. 370-375, 1958.

[20] A. J. Rowan and E. Tolunsky, Primer of EEG: With a Mini-Atlas. USA: Elsevier Science, 2003.

[21] M. F. Bear, B. W. Connors, and M. A. Paradiso, "Rhythms of the Brain," in Neuroscience: exploring the brain, 2nd ed, M. F. Bear, B. W. Connors, and M. A. Paradiso, Eds. USA: Lippincott Williams \& Wilkins, 2001, pp. 606-636.

[22] J. Malmivuo and R. Plonsey, Bioelectromagnetism: Principles and Applications of Bioelectric and Biomagnetic Fields: Oxford University Press, USA, 1995.

[23] M. R. Neuman, "Biopotential Electrodes," in Medical Instrumentation: Applicantion and Design, 3rd ed, J. G. Webster, Ed. USA: John Wiley and Sons, 1998.

[24] B. B. Winter and J. G. Webster, "Reduction of Interference Due to Common Mode Voltage in Biopotential Amplifiers," IEEE Transactions on Bioimedical Engineering, vol. 30, pp. 58-62, 1983.

[25] Analog Devices, "Low Cost, Low Power Instrumentation Amplifier - AD620," Analog Devices, 1999.

[26] D. J. Comer and D. T. Comer, Advanced Electronic Circuit Design. Great Britain: Wiley, 2003.

[27] B. B. Winter and J. G. Webster, "Driven-Right-Leg Circuit Design," IEEE Transactions on Bioimedical Engineering, vol. 30, pp. 62-66, 1983.

[28] Texas Instruments, "SimpliciTI Network Protocol," 2008.

[29] D. F. Dinges, "An overview of sleepiness and accidents," Journal of Sleep Research, vol. 4, pp. 4-14, 1995.

[30] National Transport Commission (NTC), "Fatigue Management Within The Rail Industry: Review of Regulatory Approach," Melbourne, Australia 2004.

[31] M. Ashiya, S. Sone, Y. Sato, and A. Kaga, "Application of pure electric braking system to electric railcars," in Proceedings of 6th International Workshop on Advanced Motion Control, 2000, pp. 163-168.

[32] A. Austin and P. D. Drummond, "Work problems associated with suburban train driving," Applied Ergonomics, vol. 17, pp. 111-116, 1986.

[33] A. Jabez, "Back in control," Nursing Times, vol. 89, pp. 46-47, 1993.

[34] G. J. S. Wilde and J. F. Stinson, "The monitoring of vigilance in locomotive engineers," Accident Analysis \& Prevention, vol. 15, pp. 87-93, 1983.

[35] G. D. Edkins and C. M. Pollock, "The influence of sustained attention on Railway accidents," Accident Analysis \& Prevention, vol. 29, pp. 533-539, 1997.

[36] G. D. Roach, J. Dorrian, A. Fletcher, and D. Dawson, "Comparing the effects of fatigue and alcohol consumption on locomotive engineers' performance in a rail simulator," Journal of Human Ergology, vol. 30, pp. 125-130, 2001. 
[37] RailCorp, "Careers in Service Delivery," 2008.

[38] T. Åkerstedt and P. M. Nilsson, "Sleep as restitution: an introduction," Journal of Internal Medicine, vol. 254, pp. 6-12, 2003.

[39] B. T. Jap, S. Lal, P. Fischer, and E. Bekiaris, "Using EEG spectral components to assess algorithms for detecting fatigue," Expert Systems with Applications, vol. 36, pp. 2352-2359, 2009.

[40] H. Tietze, "Stages of wakefulness during long duration driving reflected in alpha related events in the EEG," in Proceedings of the International Conference on Traffic and Transport Psychology ICTTP, Bern, Zwitserland, 2000.

[41] T.-P. Jung, S. Makeig, M. Stensmo, and T. J. Sejnowski, "Estimating alertness from the EEG power spectrum," IEEE Transactions on Bioimedical Engineering, vol. 44, pp. 60-69, 1997.

[42] A. Subasi, "Automatic recognition of alertness level from EEG by using neural network and wavelet coefficients," Expert Systems with Applications, vol. 28, pp. 701-711, 2005.

[43] M. K. Kiymik, M. Akin, and A. Subasi, "Automatic recognition of alertness level by using wavelet transform and artificial neural network," Journal of Neuroscience Methods, vol. 139, pp. 231-240, 2004

[44] C.-T. Lin, R.-C. Wu, S.-F. Liang, W.-H. Chao, Y.J. Chen, and T.-P. Jung, "EEG-based drowsiness estimation for safety driving using independent component analysis," IEEE Transactions on Circuits and Systems I: Regular Papers, vol. 52, pp. 2726- 2738, 2005.

[45] A. Williamson and T. Chamberlain, "Review of onroad driver fatigue monitoring devices," New South Wales, Australia: NSW Injury Risk Management Research Centre, University of New South Wales, 2005.

[46] J. Yao, R. Schmitz, and S. Warren, "A Wearable Standards-Based Point-of-Care System for Home Use," in international Conference ofthe IEEE EMBS, Cancun, Mexico, 2003.

[47] C.-H. Shih, K. Wang, and H.-C. Shih, "An adaptive bluetooth packet selection and scheduling scheme in interference environments," Computer Communications, vol. 29, pp. 2084-2095, 2006.

[48] H. A. Thompson, "Wireless and Internet communications technologies for monitoring and control," Control Engineering Practice, vol. 12, pp. 781-791, 2004.

[49] I. Chlamtac, M. Conti, and J. J. N. Liu, "Mobile ad hoc networking: imperatives and challenges," Ad Hoc Networks, vol. 1, pp. 13-64, 2003.

[50] N. Wang, N. Zhang, and M. Wang, "Wireless sensors in agriculture and food industry--Recent development and future perspective," Computers and Electronics in Agriculture, vol. 50, pp. 1-14, 2006.

\section{Biography}

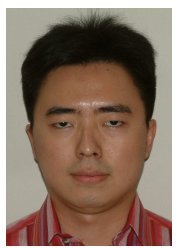

Surya Darma Ridwan, BEng (Electrical), MBA, graduated with a Bachelor degree in Electrical Engineering from Nanyang Technological University, Singapore. His final year thesis was on the implementation of multi-carrier CDMA communication protocol on DSP platform. Upon graduation, Surya worked for Philips Electronics Singapore as an Electronic Design Engineer. $\mathrm{He}$ was in charge of Digital LCD TV. He pursued his MBA degree at University of Technology, Sydney.

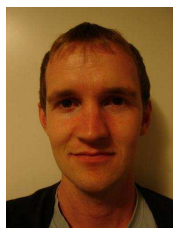

Robert Thompson is studying at the University of Technology, Sydney for a Bachelor of Electrical Engineering. Whilst studying Robert has gained experience in both the biomedical and mining industries and received the 2006 Institute of Instrumentation, Control \& Automation Electrical Student Award. Robert's interests include medical instrumentation, control theory, power electronics, and electrical machinery.

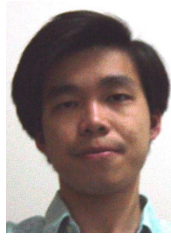

Budi Jap, BEng (Computer System), BA (International Studies), is currently completing his $\mathrm{PhD}$ at University of Technology, Sydney (UTS), Australia. The main research area is in driver fatigue and finding the means to detect driver fatigue from different physiological signals, such as brain or heart activities.

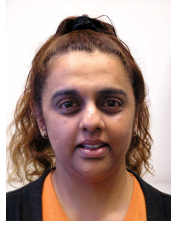

Dr Sara Lal, (PhD, MAppSc, GCHE, DipLaw) is an academic at the University of Technology, Sydney. Some of Dr Lal's areas of research are neuroscience, cardiovascular, sleep disorder, cognitive function, transportation safety, and sensor and technology development. Dr Lal has attracted various competitive grants and has published a book, book chapters, refereed journals and conference papers.

Peter Fischer, BEng (Electrical), Fellow of the Institute of Engineers, Australia. Director of Signal Network Technologies Pty Ltd. Researching and developing railway safety and driver vigilance systems for over 40 years. Expert in physiological and physical monitoring fields. 